\title{
CEZEAU : un projet de banque de terminologie en mécanique des sols
}

\section{Les problèmes de terminologie}

S'il existe aujourd'hui dans le monde un nombre important (et bien entendu croissant) de banques de données terminologiques, c'est bien sûr grâce à l'impulsion initiale donnée par des pays où les problèmes de langues demandaient des solutions rapides tant ils étaient chargés de conséquences pour l'économie ou la politique de ces pays.

L'Annuaire des Banques de Données Françaises que doit publier prochainement la Mission Interministérielle de I'Information Scientifique et Technique (M.I.D.I.S.T.) montrera exactement quelles sont les réalisations françaises dans ce domaine, mais on sait déjà qu'elles sont peu nombreuses. L'article du Monde du 23 juin 1982 soulignait cette situation à l'occasion du dernier Colloque International de la Francophonie à Bruxelles: "l'absence de la France sur le front de la terminologie informatisée [...] étonne toujours les francophones hors de l'hexagonen.

Mais cette absence n'est pas totale. Certains secteurs veillent en fait de très près sur leur terminologie sachant que, pour certains d'entre eux, la survie est à ce prix : $50 \%$ de la terminologie de certains secteurs de l'électronique a changé en cinq ans, et l'industrie du bâtiment a créé au sein de la Commission Économique Européenne un groupe de travail chargé d'étudier ses problèmes de terminologie.

La Mécanique des Sols ne pose pas, sans doute, de problèmes terminologiques aussi urgents. D'une part son vocabulaire n'a certainement pas évolué au même rythme que celui de l'électronique et d'autre part les mots et expressions techniques de la Mécanique des Sols ont été et restent très largement contrôlés grâce à d'excellents dictionnaires imprimés.

Mais (et c'est un lieu commun de le souligner) nous sommes à l'âge de l'ordinateur et nous pouvons nous interroger sur les conséquences (graves sans doute) que pourrait avoir une attitude immobiliste sur les problèmes de terminologie dans ce secteur alors que d'autres domaines et d'autres pays sont déjà en train d'explorer la voie et de prendre la tête. Quatre points paraissent constituer de bonnes raisons d'organiser la terminologie en Mécanique des Sols à l'aide de méthodes informatiques :

1) La nécessité de disposer d'une banque constamment tenue à jour et capable de rendre compte de l'évolution de la terminologie sur le plan international.

2) La possibilité d'utiliser un outil infiniment plus puissant que le papier imprimé et capable, outre les facilités de mise à jour, de stocker sous les formes les plus diverses un ensemble important d'informations sur les notions.

3) La nécessité de disposer d'un outil capable à la fois d'organiser le domaine terminologique français de la Mécanique des Sols et d'assurer l'interface avec l'anglais (et d'autres langues), ceci dans le double but de conserver l'intégrité du français scientifique et de tenir compte de l'importance de l'anglais dans la communication internationale.

4) L'intérêt que présente un organisme spécialisé en matière de terminologie capable de fournir des informations dont la crédibilité est assurée par les rapports entretenus par cet organisme avec les spécialistes du domaine et les instances internationales de la terminologie.

\section{Le Projet CEZEAU}

Le programme CEZEAU en cours de réalisation à Clermont-Ferrand vise à la construction d'une banque de données terminologiques en Mécanique des Sols, connectable sur les réseaux existants et consultable en ligne et en batch.

Il est mis en œuvre par le Laboratoire de Recherches 
en Informatique de l'Université de Clermont-Ferrand : section "étude du langage et banques de données terminologiques " (1).

L'équipe de CEZEAU est interdisciplinaire. Elle travaille en association avec I'U.M.I.S.T. de Manchester (University of Manchester Institute of Science and Technology), l'organisation internationale IN. FO. TERM (Information in Terminology) de Vienne et est membre du réseau TermNet (Terminology Network).

Elle fait doublement appel aux spécialistes de Mécanique des Sols. Au niveau local, le Laboratoire de Mécanique des Sols de Clermont-Ferrand assure la validation des données mais il va de soi qu'il appartient aux instances nationales de la Mécanique des sols de contrôler le vocabulaire du domaine. De même, les spécialistes et utilisateurs de vocabulaires de Mécanique des Sols sont-ils instamment invités à faire connaître leurs opinions et à participer au projet. Enfin, le système est conçu pour tenir compte des avis des utilisateurs.

\section{Principes de fonctionnement}

Les services rendus par CEZEAU sont ceux d'une banque de terminologie classique, mais bien que bilingue, CEZEAU n'est pas spécialement conçue pour la traduction comme le sont un grand nombre de banques actuelles. CEZEAU s'adresse en fait à l'ensemble des utilisateurs de vocabulaires et de notions de Mécanique des Sols.

II est prévu que CEZEAU soit relié aux réseaux existants et notamment devienne serveur (en ligne) dans le réseau EURONET. La banque pourra fournir en outre des informations sélectives et produire des glossaires.

\section{Fiche terminologique}

CEZEAU donnera sur l'entrée (terme) proposée par l'utilisateur et sur la notion à laquelle elle renvoie au minimum les renseignements suivants en anglais ou en français.

1 Renseignements généraux (techniques)

- La norme associée au terme (normes A.F.N.O.R. I.S.O., etc.).
- La date de l'enregistrement (âge du terme dans la banque).

- Le code C.D.U. du domaine (code dans le système de Classification Décimale Universelle).

- Les sources de l'enregistrement (il s'agit des textes disponibles ou non en mémoire, dans lesquels le terme a été trouvé).

\section{Renseignements relatifs à la notion}

- Équivalents de l'entrée :

- équivalents on anglais ou vice versa (exemple argile $=$ clay)

- synonymes et commentaire sur l'emploi (exemple dame à explosion = grenouille : terme familier).

- Relations hiérarchiques avec d'autres notions (exemple : argile est un type de sol, les types de sols sont argile, marne, sable, etc. De la même façon, argile active est un type d'argile, etc.).

- Définition de la notion.

\section{Renseignements linguistiques}

- Indication grammaticale (nom, verbe, adjectif, adverbe).

- Expressions plus grandes que l'entrée et contenant celle-ci (exemple : argile/argile active/activité des argiles, etc.).

- Déroulement de la liste des termes de la banque alphabétiquement dans les deux sens (comme on feuillette un dictionnaire classique).

- Contextes linguistiques contenant l'entrée (il est possible à l'utilisateur d'obtenir un certain nombre de contextes linguistiques pouvant l'éclairer sur les utilisations du terme auquel il s'intéresse).

\section{État du projet}

Une maquette est en cours de construction et des démonstrations pourront être faites dans le courant de 1983 portant sur un nombre réduit de champs : équivalents en langue étrangère, synonymes, définitions, listage du contenu de la banque.

La banque CEZEAU elle-même pourrait être opérationnelle en 1985. 